\title{
¿EDUCACIÓN INCLUSIVA EN NUESTROS CENTROS EDUCATIVOS? SÍ, PERO ¿CÓMO?
}

\author{
José Ma Fernández Batanero \\ Universidad de Sevilla
}

\begin{abstract}
RESUMEN: Desde su origen hasta hoy la Educación Inclusiva ha ido ganando terreno como movimiento que desafía y rechaza las políticas, culturas y prácticas educativas que promueven cualquier tipo de exclusión. A la vez la inclusión educativa ha ido abriendo caminos que tienen como meta una educación eficaz para todos los alumnos. En el presente artículo reflexionamos sobre algunos de los cambios que los centros educativos tendrán que afrontar si quieren plantearse como meta esta modalidad de educación.

SUMMARY: From its origin to today the Inclusiva Education has been taking terrain like movement that defies and rejects the policies, cultures and educative practices that promote any type of exclusion. Simultaneously the educative inclusion has been laying ways that they have as it puts an effective education for all the students. In the present article we reflected on some of the changes that the educative centers will have to confront if they want to consider as it puts this modality of education.
\end{abstract}

PALABRAS CLAVES: inclusión, estrategias, curriculum, organización.

KEY WORDS: inclusion, strategies, curriculum, organization.

\section{INTRODUCCIÓN}

La integración escolar se caracterizó por plantear el reto de atender en la escuela ordinaria a los alumnos para los que era necesaria una respuesta diferente y que eran encaminados hacia otro tipo de centros; por pretender que todos los alumnos, con los recursos necesarios, pudieran alcanzar los objetivos establecidos con carácter general. Por su parte la educación inclusiva implica que todos los niños y niñas de una determinada comunidad aprendan juntos independientemente de sus condiciones personales, sociales o culturales, incluidos aquellos que presentan discapacidad (Stainback, Stainback y Jackson (1999). Es decir, se pretende una escuela que haga efectivo el derecho a la educación, la igualdad de oportunidades y la partici- 
pación, ya que no pone requisitos ni mecanismos de entrada, selección o discriminación de ningún tipo, caracterizada por la convivencia en igualdad y diversidad, de tal forma que se le ofrezca a cada uno lo que necesite para el desarrollo de sus potencialidades. Reconocer la igualdad entre las personas implica una determinada concepción del mundo, de los seres humanos y de los valores que han de usarse como referente a la hora de convivir.

Durante las tres últimas décadas del siglo pasado se han abierto nuevos caminos para trazar rutas alternativas (principio de normalización, informe Warnock, integración...). Atrás quedó ya la pedagogía de la exclusión, basada fundamentalmente en la idea de que estudiar era un privilegio, y que los alumnos mal educados o con problemas en su aprendizaje debían dejar las escasas plazas escolares a otros mejor dotados.

Hemos pasado de una escolarización escasa y llena de lagunas, a una tasa de escolarización del $100 \%$ en educación primaria y del $90 \%$ en secundaria. Este hecho supone cambios importantes en la manera de entender y concebir la educación.

Estamos asistiendo al crecimiento de la idea de conseguir una "educación abierta para todos". Muchas son sus denominaciones: educación inclusiva, educación integradora, educación en la diversidad, educación para todos, atención a la diversidad, etc. Todos estos términos mantienen una idea común: responder a las necesidades de todos los alumnos, con independencia de su naturaleza o grado de necesidad que presenten. Una educación sin exclusiones, en la que convivan y aprendan alumnos de distintas condiciones sociales, de diferentes culturas y distintas capacidades e intereses, desde los más capaces hasta los que tienen alguna discapacidad. Esta nueva educación es, por tanto, para todos y cada uno de los niños que llegan, saben y viven su propia realidad, ha de acoger a cada niño con lo que trae y lo que es, ni más ni menos. Hoy día, sabemos que cada persona somos una de las infinitas combinaciones entre rasgos físicos, psicológicos, sociales, culturales... todos somos diferentes, igual de válidos y con los mismos derechos.

Significa esto que la educación inclusiva no está destinada a los alumnos con necesidades especiales exclusivamente, sino a todos los niños que quieran pertenecer a esa escuela: alumnos con deficiencias y sin ellas, con dificultades de aprendizaje y sin ellas, alumnos de diferentes culturas, alumnos que provienen de familias con diferentes niveles socioeconómicos, alumnos con diferentes sistemas de valores, distintas religiones...

En esencia, como sostiene Ainscow (2001) una educación de orientación inclusiva pretende disminuir todas las barreras al aprendizaje y a la participación, independiente de quién las experimenta y dónde se encuentren estas barreras, en las culturas, políticas y prácticas de una escuela. Se enfatiza la movilización de recursos infrautilizados a nivel de personal docente, alumnos, consejo escolar, padres y otros integrantes de las comunidades escolares. En este contexto, la diversidad es considerada un rico recurso de apoyo al aprendizaje y a la enseñanza.

Actualmente, según se desprende del informe de 2003 sobre necesidades educativas especiales en Europa elaborado por la Agencia Europea para el Desarrollo de la Educación Especial, la tendencia actual en la Unión Europea y en los países candidatos es desarrollar políticas dirigidas hacia la educación inclusiva, proporcio- 
nando al profesorado varios tipos de apoyo tales como personal complementario, materiales, cursos de formación y equipamiento.

\section{Cómo lleVAr la inCluSión a la PRÁCtica eduCATiva}

Educar en este contexto no es tarea fácil, ya que implica un cambio profundo y esencial en los Proyectos Educativos de los centros docentes, de forma que contribuya a reconsiderar su organización, curriculum y servicios entre otros aspectos. Y ello, es preciso que se lleve a cabo en el marco de principios básicos como: 1. La participación, 2. Las expectativas positivas, 3. Enseñanza y aprendizaje interactivo, y 4. El apoyo a los profesores.

Figura 1. Cambios en el Proyecto Educativo.

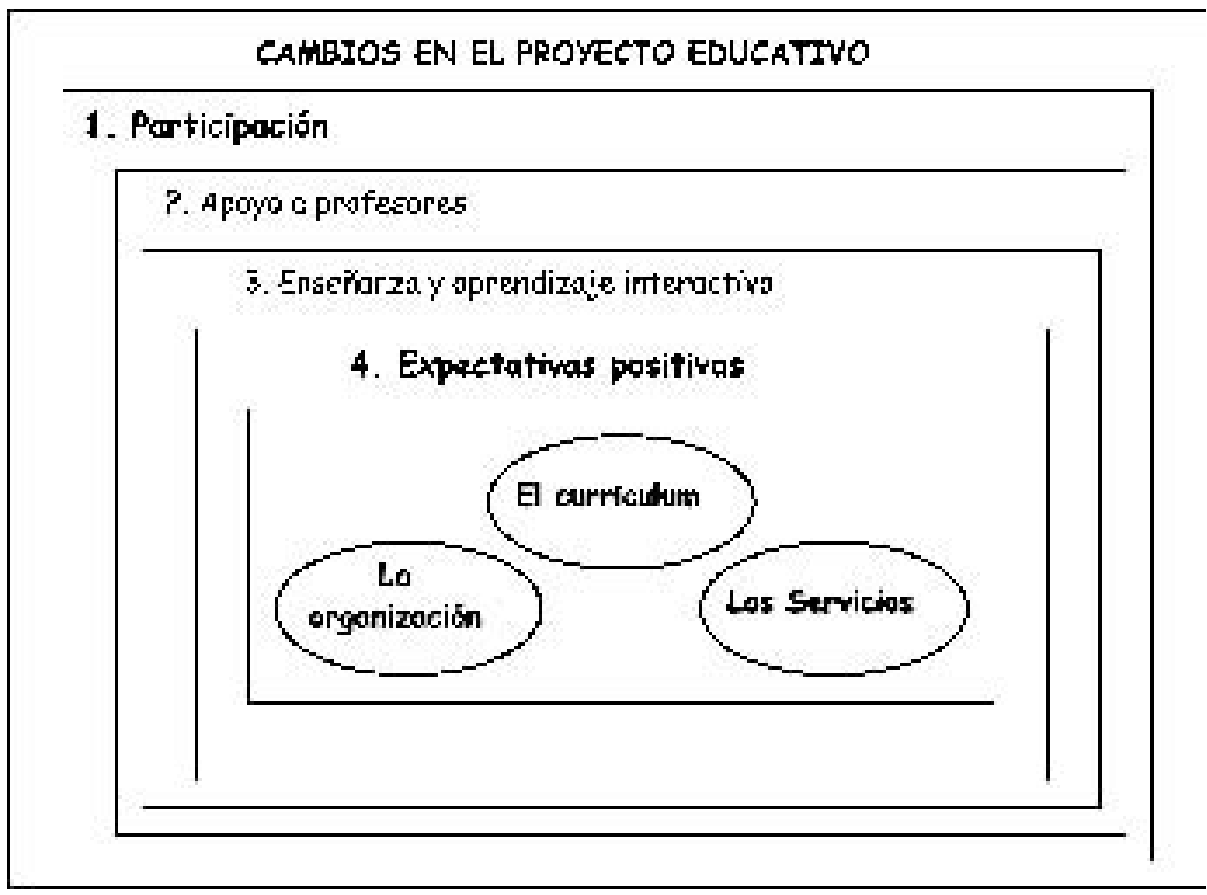

Esta transformación ha de comenzar desde los cimientos, desde las estructuras más profundas para que realmente sea un renacer. Esto no significa que todo lo que se ha conseguido hasta ahora no sea válido. En absoluto, debemos quedarnos con lo que nos sirve para despojarnos sin temor de lo que hemos de cambiar y así lanzarnos de lleno a la tarea de la construcción de esa escuela de calidad para todos los alumnos (Pascual, 2004).

Las escuelas de orientación inclusiva deben iniciar un proyecto de transformación social y cultural del propio centro, siendo imprescindible para ello que se tengan en cuenta tres premisas fundamentales: 
- Un proyecto común en el que participe toda la comunidad educativa.

- Un proyecto educativo que, en base a la diversidad de su propia realidad educativa, contemple una nueva forma de organización, de concebir el curriculum y los servicios educativos.

- Un proyecto impregnado por una serie de valores éticos.

\subsection{Un proyecto común en el que participe toda la comunidad educativa}

Una educación sin exclusiones, en la que convivan y aprendan alumnos de distintas condiciones sociales, de diferentes culturas y distintas capacidades e intereses, desde los más capaces hasta los que tienen alguna discapacidad pasa necesariamente por considerar el aprendizaje como una tarea de todos. El aprendizaje escolar no puede quedar sólo en manos de las maestras y los maestros, sino que debe estar abierto a la participación todos los agentes educativos posibles: profesorado, familia, voluntariado, instituciones y asociaciones del barrio. Todos comparten la meta global de la formación y todos participan activamente en la planificación, realización y evaluación de las actividades del centro. En este sentido, se debe optimizar la utilización de los recursos del barrio y de la comunidad mediante la planificación y la actividad conjunta.

Los Consejos Escolares, los equipos directivos y las comisiones gestoras deben asumir el papel de la gestión y la coordinación del proyecto por encima de la dirección unipersonal. Es necesario crear comisiones mixtas para coordinar todo el trabajo, delegando responsabilidades, donde el profesorado asume un nuevo papel de dinamizador y coordinador de quienes colaboran en sus tareas.

De este modo, la concreción de las finalidades educativas supone una tarea de toda la comunidad. En conclusión, las Finalidades Educativas requieren una reflexión ética indisociable de una explicitación de valores deseables. Además, permiten planificar las líneas de actuación del Centro, dado que esta concreción debe encontrarse en el Proyecto Curricular y en el reglamento de Organización y Funcionamiento.

Uno de los peligros implícitos en la elaboración de las Finalidades Educativas consiste en convertirlas en un ejercicio mecánico más para dar cumplimiento burocrático a una exigencia de la Administración Educativa. Un mecanismo que evita esta situación es lo que Bolivar (1993) denomina: "priorización de ámbitos concretos de mejora". Este mecanismo consiste en decidir de forma realista qué necesidades son las más prioritarias, en función del problema detectado. De esta forma y para una mejor comprensión y tratamiento de las Finalidades Educativas, éstas podrían agruparse en los siguientes ámbitos: finalidades de ámbito convivencial, pedagógico, administrativo y organizativo.

\subsection{Un proyecto educativo que, en base a la diversidad de su propia realidad educativa, contemple una nueva forma de organización, de concebir el curriculum y los servicios educativos}

La organización es el escenario que alberga las actividades y en el que se desarrolla la convivencia. Es, también, el requisito que hace viable llevar a la práctica 
los postulados que pretenden conjugar el respeto a la igualdad y la necesaria atención a la diversidad.

Durante muchos años y todavía hoy la organización predominante en nuestras escuelas es aquella que facilita un proceder homogéneo: los alumnos y alumnas son distribuidos en grupos de edad (como si todos los que tienen las misma edad tuvieran las mismas capacidades y destrezas); se pone en marcha una metodología idéntica para todo el grupo; se hacen módulos horarios iguales para todos, asumiendo que todos los alumnos deben acabar las tareas a la vez; se supone que un año escolar debe tener la misma duración para todos los aprendices; los profesores utilizan baremos parecidos para calificar a todos los chicos de la misma edad y curso, sin tener en cuenta cual ha sido su punto de partida al comienzo del año; incluso la legislación es la misma y ha de ser cumplida en el mismo tiempo y de la misma forma para todos los centros.

Frente a ese proceder homogéneo se hace necesario plantear un enfoque diferente de la práctica educativa. Una organización sensible con la diversidad debe cumplir una serie de exigencias: flexibilidad (la rigidez impide realizar cambios y adaptaciones con el ritmo y la profundidad conveniente); permeabilidad (posibilidad de penetración en los dos sentidos, desde el centro educativo al entorno y desde el propio entorno al centro); creatividad (para favorecer la innovación) y colegiabilidad (un proyecto que se refiere a toda la institución, no a cada profesor de manera aislada).

Y para ello, es necesario que se desarrollen algunas estrategias básicas de organización que resultan útiles a la hora de responder a las necesidades de todos los alumnos, por parte de los centros educativos, como son:

- Formación de grupos interactivos. Los grupos interactivos consisten en organizar el aula de manera que haya pequeños grupos heterogéneos de 4 o 5 alumnos que, en colaboración, resuelven tareas de clase con la supervisión de una persona adulta (familiar, voluntario, etc.) que está dentro del grupo. El ritmo de trabajo es alto y, además, solidario. La tarea del profesor o profesora es la coordinación de la clase. La composición de estos grupos favorece la mezcla de niveles, de manera que en la elaboración de las tareas de clase, los alumnos colaboran en su resolución. De esta forma, los alumnos que han adquirido un aprendizaje lo refuerzan explicándolo a los que todavía no lo han hecho, que lo entienden mejor confrontando la explicación de los educadores y la de los compañeros.

- Creación de espacios de trabajo polivalentes.

Todos los espacios del centro deben estar dispuestos de forma que el alumno pueda realizar con soltura todas las actividades encaminadas al desarrollo personal, al aprendizaje y a la convivencia, siendo agradable y placentero ya que el alumno ha de permanecer en su interior una gran parte del día y de su vida. Como apunta Muntaner (1998, 57): El espacio del aula debe organizarse al servicio de las pretensiones educativas y debe ser un elemento favorecedor de la flexibilidad y de la diversidad en la oferta de actividades del 
alumno, no puede convertirse en un corsé que limite las posibilidades de actuación del grupo clase.

- Planificación de tiempos flexibles (horarios a la carta).

El horario escolar ha de ser cambiado, si queremos que los centros y los profesores puedan mejorar la calidad de la enseñanza. Por tanto, ante la demanda de horarios rígidos y cerrados, el centro ha de defender modelos integradores del curriculum, flexibles, etc. que no pasen normalmente por la adscripción rígida de horas a cada materia en franjas de horario inamovibles.

El criterio de flexibilización ha de permitir organizar variaciones en los horarios y en su distribución, siendo necesario, para ello, entablar discusiones en los propios centros, donde los criterios de partida pueden ser (Masalles y Rigol (2000): estar el máximo de horas con el tutor o la tutora; distribución de las materias en las horas sin primar unas sobre otras: todas las materias son igual de importantes; adecuación en la distribución de las áreas y ritmos a lo largo de un día cualquiera; módulos horarios diversos (evitar la idea de sesiones de una hora por sistema) buscando ratos largos que permitan una mayor flexibilidad y el desarrollo de un proceso de aprendizaje más adecuado; combinar las actividades que se realizan a lo largo del día con la distribución de las horas con criterios de tipo metodológico; combinar en el horario las horas dedicadas a materias o áreas, con las actividades (rincones, proyectos, etc.).

Decidir en última instancia en función de criterios pedagógicos y en relación con las necesidades de los niños y niñas, más que con criterios administrativos o de necesidades de maestros o maestras.

- Establecimiento de agrupamientos flexibles.

Uno de los aspectos organizativos que tienen más incidencia en la creación de un curriculum, que tenga en cuenta a todos los alumnos, es el referente a los agrupamientos flexibles. Esta modalidad de agrupamiento tiene como objetivo favorecer la atención a las diferencias individuales desde la adaptación del proceso de enseñanza-aprendizaje a los intereses y ritmos de aprendizaje de cada alumno y, en consecuencia, organizar la dinámica de la clase según esta variable. Desde la perspectiva organizativa, los agrupamientos flexibles no sólo afectan a los alumnos y alumnas, sino que también condicionan la programación curricular, así como la organización del trabajo de los profesores, e incluso la de los tiempos y los espacios (Albericio, 1994).

- Tutorías individualizadas.

La diversificación del sistema de tutorías es una condición imprescindible para realizar una atención verdaderamente individualizada. En nuestro curriculum actual las tutorías tienen un carácter grupal. Esto exige una modificación de la estructura temporal en la atención a los alumnos y alumnas y familias. Como sostienen Hargreaves, Earl y Ryan (1998) al afirmar que muchos jóvenes no adquirirán los niveles básicos académicos si no les preocupa y atienden a lo que aprenden. Perderán la vinculación con su aprendizaje si se sienten aislados, perdidos y desatendidos en el aula. 
- Dotación de servicios de apoyo al centro.

El centro es el responsable de encontrar la respuesta a las necesidades de todos los alumnos. Todo el profesorado debe encontrarse implicado en el reto de: analizar las necesidades educativas derivadas de cada contexto de intervención (a nivel de centro, de aula e individualmente), adecuar la respuesta a dichas necesidades y hacer el seguimiento y evaluación de su oferta educativa.

Con respecto al currículum éste debe ser abierto y dinámico en función de las necesidades y flexible a los cambios sociales, metodológicos y estructurales. Un currículo que garantice, en los alumnos, el desarrollo de las competencias necesarias para desenvolverse en la sociedad actual. Esto implica dejar atrás la imagen de las clases frontales centradas en la enseñanza, carentes de sentido y descontextualizadas de la realidad para dar paso a un nuevo proceso a través del cual los alumnos tienen la posibilidad de compartir y disfrutar en un clima cálido y acogedor situaciones de aprendizaje relacionadas con sus propias experiencias, en contextos donde se valoran, toman en cuenta y respetan sus diferencias individuales y en el cual existe cotidianamente la oportunidad de beneficiarse junto con los demás a través de los trabajos cooperativos y de la formación de grupos heterogéneos.

Para ello, es necesario pasar de un currículo estándar y fragmentado en materias a un currículo diversificado, multicultural, interdisciplinar y funcional. La educación diversificada no es una idea nueva, sino una lógica comprensión sobre los modos en los que los niños aprenden. Si sabemos que cada uno aprende de forma diferente, tendremos que diversificar el curriculum. Diversificar es adaptar, adecuar la enseñanza a los intereses de cada alumno. La creciente diversidad racial y étnica de nuestra sociedad obliga a que los currículos escolares tengan en cuenta las diferencias entre los niños. Un currículo interdisciplinar y temático se adapta mucho mejor que las prácticas tradicionales de lecciones magistrales, trabajos en el pupitre y enseñanza basada en el libro de texto.

Cuando el contenido del currículo se organiza de forma interdisciplinar es más significativo y estimula más a los alumnos.

El Ilamado curriculum funcional pone su mayor énfasis en que prepare para la vida y no sólo para las distintas etapas académicas. La noción de funcionalidad implica la utilidad de algo o la utilidad para algo.

La puesta en práctica de un curriculum que tenga en cuenta a todos los alumnos, implica, entre otras, necesariamente la consideración de una serie de estrategias de enseñanza. Fomentar la participación del alumno: motivar, utilizando como referencia los intereses vitales de los alumnos; encontrar sentido al trabajo que el alumno desarrolla en el aula; hacer que la enseñanza y el aprendizaje sean como la vida real; tomar decisiones en torno a los objetivos, contenidos, actividades, materiales y la evaluación; utilizar técnicas de trabajo individual y de grupo; usar y adaptar la tecnología; crear y adaptar materiales y recursos variados; organizar el trabajo fuera del aula.

\subsection{Un proyecto impregnado por una serie de valores éticos}

La educación en valores, que se justifica por la necesidad de una serie de principios éticos que nos sirvan para evaluar nuestras acciones y las de los demás. 
La investigación sobre escuelas realmente excelentes ha demostrado que, a diferencia de aquellas otras en las que prevalecen las normas individuales, se han esforzado en construir el norte de su misión educativa, una cultura compartida y un conjunto de normas comunes respecto a la enseñanza y la formación (Coleman y Hoffer, 1987).

El trabajo pedagógico sobre procedimientos, actitudes y valores se presenta como una urgencia pedagógica ante una sociedad en la que los grandes problemas de la humanidad y los principios que regulan las relaciones entre los hombres, las mujeres y los pueblos, y las relaciones de estos con su entorno natural, requieren reorientaciones éticas y morales y no tanto soluciones técnicas o científicas (GerviIla, 1997). En este sentido, se pide que la educación obligatoria capacite a los alumnos y alumnas para desenvolverse en la sociedad haciendo pleno uso de sus derechos y haciendo frente a sus responsabilidades, es decir, dotarles de los conocimientos necesarios para entender la realidad y poder actuar sobre ella, pero ello supone también educarles en ciertas actitudes ante los problemas sociales (Actitudes de defensa, protección, conservación y mejora del medio ambiente y la calidad de vida dentro de él; actitudes de respeto, tolerancia, justicia ante problemas de violencia, agresión, etc.; actitudes responsables y críticas ante todo tipo de sugerencias o de incitaciones al consumo que pueda limitar, perjudicar o empobrecer la salud y la vida personal o colectiva...). En definitiva, se trata de formar ciudadanos activos, solidarios y abiertos al mundo, que valoren la relación entre sus acciones, los resultados y las consecuencias previsibles, autónomos, capaces de deliberar, juzgar y escoger sobre la base de sus propias decisiones.

Se impone otra nueva educación, que responda a otra concepción del ser humano, una educación que restaure los valores éticos esenciales, donde prime la adquisición de hábitos de convivencia, de solidaridad, de justicia y de respeto para nuestros semejantes. Es el cambio en las actitudes el que nos Ilevará irreversiblemente a una sociedad más humana y solidaria.

Educar en valores significa propiciar el establecimiento de un sistema coherente de valores y, mucho más aún, lograr un acuerdo generalizado al respecto, pese a lo cual es imperativo su búsqueda con todas nuestras fuerzas y voluntad si queremos un mundo equilibrado, solidario y en paz. Debemos de desechar la famosa frase de "Haced lo que yo diga pero no hagáis lo que yo hago", ya que constituye la expresión del testimonio contradictorio en las actuales sociedades de nuestros adultos. Esto significa alcanzar un compromiso de trabajo entre profesores y padres, y estos con la comunidad.

Los valores a conseguir constituyen un aspecto muy importante en la enseñanza. Los docentes y estudiantes deben de esforzarse para lograr hábitos de trabajo que, practicados de forma regular, produzcan resultados beneficiosos y motivantes para todos los alumnos.

Con las normas podemos concretar la forma de convivencia que nos proponemos. Algunas características serían: 
Cuadro no 1. Características de las normas

- Tendrán un objetivo claro y serán flexibles con las pequeñas variaciones en ellas.

- Serán negociadas y elegidas en base a alguna razón, sentido o intención.

- Se aprobarán por todo el alumnado y el profesorado del curso y, en lo posible, con la implicación de las familias.

- Será preferible que sean pocas pero que se lleven a cabo.

- Poseerán un lenguaje incentivador y no prohibitivo.

- Reforzarán las conductas positivas.

- Se basarán en principios de convivencia y no en un listado de posibles sanciones.

- Tenderán hacia la responsabilización colectiva y compartida, evitando la huida de los conflictos.

- Serán una vía de resolución de los problemas que puedan surgir.

- Tendrán en cuenta a todo el alumnado.

- Se referirán a cada uno de los espacios del centro (aulas, pasillos, patios, biblioteca, etc.) y tiempos (horas de clase, recreos, etc.).

- Incluirán en ellas una organización del espacio y tiempo del alumnado, así como posibles actividades y valoración de las mismas.

\section{A MODO DE REFLEXIÓN}

Poner en práctica una educación donde todos los alumnos sean atendidos, donde se les dé respuesta a todas sus necesidades, donde estos se sientan partícipes, y donde la eficacia sea una característica común, no está exenta de problemas y comentarios. Para muchos docentes, ello no es más que pura ciencia ficción. Muchos todavía piensan que es parte de la mucha teoría que se les enseña a los futuros profesores en su proceso de formación permanente. Pero a pesar de todo, la realidad educativa nos demuestra que es posible una verdadera enseñanza eficaz para todos los niños y niñas de una comunidad. Un ejemplo de ello, lo podemos observar en el modelo de comunidades de aprendizaje, donde el resultado positivo es evidente. Resultado fruto de un proceso que tiene su punto de partida en la sensibilización de la propia comunidad, para pasar posteriormente a tomar decisiones, a seleccionar las prioridades, a planificar, a investigar, a formar, a evaluar los resultados, etc., en definitiva a participar con mayúsculas. No es más que intentar crear la escuela que soñamos para nuestros hijos. Para muchos una utopía, pero sin olvidar que las utopías han sido el motor de todos los cambios que se han producido en la humanidad. En lo posible tenemos que recuperar el valor de la utopía como motor de transformación de la sociedad ¿Acaso no fue la utopía la que sirvió para eliminar la esclavitud?

Por qué no creer en clases multiedad al lado de clases de estudiantes del mismo grado, por qué no creer en la resolución de conflictos, en la eficacia de los grupos cooperativos, en el papel de los mediadores, en los estudiantes comprometidos y motivados, en un verdadero apoyo curricular, en los maestros motivadores, etc. ¿Por qué no?

Mientras la utopía educativa la tengamos en nuestro horizonte, podremos recorrer el camino que nos separa hacia ella. Camino fruto de la reflexión y de la prác- 
tica educativa, donde interpretemos la práctica desde la teoría e iluminemos la teoría con la propia práctica. Un camino donde la diversidad sea entendida como el punto de partida y no un obstáculo en él.

Somos conscientes que construir una escuela en la que todos quepan comienza por avanzar en la línea formativa del personal (motor del sistema) en los Centros (lugar estratégico de cambio) de cara a la construcción de Proyectos de Trabajo (PEC, PCC) y organización interna (ROF). Al igual que la integración escolar es hoy día un hecho evidente en nuestras escuelas, algún día y no muy lejano, con el esfuerzo de todos lo será la inclusión.

\section{Bibliografía}

AGENCIA EUROPEA PARA EL DESARROLLO DE LA EDUCACIÓN ESPECIAL (2003): Informe sobre Necesidades Educativas Especiales en Europa. Disponible en: http://www.european-agency.org.

AINSCOW, M. (1999): Tendiendo la mano a todos los estudiantes: Algunos retos y oportunidades. En VERDUGO ALONSO, M. A. y JORDAN, F. B. (coord.). Hacia una nueva concepción de la discapacidad. Salamanca: Amarú.

- (2001): Comprendiendo el desarrollo de escuelas inclusivas. Disponible en: http://innovemos.unesco.cl/medios/Documentos/DocumentosConsulta/epd/Ains cow2001esp.doc [Ultima consulta: 21/03/2006].

ALBERICIO HUERTA, J. J. (1994): Las agrupaciones flexibles y la escuela para el progreso continuo. Barcelona: PPV.

BOLIVAR, A. (1993): Los contenidos actitudinales en el currículo. Madrid: Escuela Española.

CARRERAS, LL., EIJO, P., ESTANY, A., GÓMEZ, Ma. T., GUICH, R., MIR, V., OJEDA, F., PLANAS, T. y SERRATS, Ma. G. (1995): Cómo educar en valores. Madrid: Narcea.

COLEMAN, J. y HOFFER, T. (1987): Public and private high scholls: The impact of communities. Nueva York: Basic Books.

DUK, C. (2000): El enfoque de la educación inclusiva. Fundación INEN.

FERNÁNDEZ BATANERO, J. Ma․ (2000): Tejiendo una tela de araña entre todos los alumnos: un currículum para la diversidad. Agenda Académica, 7 (1), 17-26.

- (2000): Una escuela para todos desde la transversalidad: los contenidos transversales. En MIÑAMBRES, A. y JOVÉ, G. (coords): La atención a las necesidades educativas especiales: de la Educación Infantil a la Universidad. Lleida: Universidad de Lleida, 493-498.

- (2001): La utilización de estrategias curriculares en la atención a la diversidad en el marco de la Educación Secundaria Obligatoria. Revista Portuguesa de Pedagogía, 35 (3), 61-80.

- (2003): Cómo Construir un currículo para todos los alumnos. De la teoría a la práctica educativa. Granada: Grupo Editorial Universitario.

- (2003): Educación Especial y Atención a la Diversidad: de la exclusión a la inclusión. Sevilla: Kronos.

- (2003): Factores de aula que pueden ayudar a todos los niños y niñas a participar en las actividades de clase. Innovación educativa, 13, 45-55. 
GERVILLA, A. (1997): Estrategias didácticas para educar en valores. Madrid: Dykinson. HARGREAVES, A., EARL, L. y RYAN, J. (1998): Una educación para el cambio. Barcelona: Octaedro.

MASALLES, J. y RIGOL, A. (2000): La organización de centros en el marco de un modelo participativo de gestión educativa. Criterios y objetivos con relación a la atención del alumnado. En ALDAMIZ ECHEVARRIA, Maㅡ M. y otros. Cómo hacerlo? Propuestas para educar en la diversidad. Barcelona: Graó.

MUNTANER, J. J. (1998): Dificultades de aprendizaje en el aula de los alumnos con Síndrome de Down. En TORRES, J. A. (dir): Intervención didáctica en educación especial. Jaén: Universidad de Jaén.

PASCUAL GARCÍA, C. (2004): Y por qué educación inclusiva. En VALLE, R. E. y DÍEZ GUTIÉRREZ, E. J. (coords). Educación y Diversidad: Comunidades Educativas: II Congreso Internacional y XXI Jornadas de Universidades y Educación Especial. León: Universidad de León.

STAINBACK, S., STAINBACK, W. y JACKSON, J. (1999): Hacia las aulas inclusivas. En STAINBACK, S. y STAINBACK, W. (coord.). Aulas inclusivas. Madrid: Narcea. 